\title{
Transgenics on the internet
}

\author{
Robert Sikorski and Richard Peters
}

Our focus this month turns to mice, specifically, internet resources for those interested in transgenic and knockout strains. We scanned over 1000 listings using our search tools and poked around the most promising leads. The results presented here are representative of the best of what is out there. Interestingly, our tools were bogged down somewhat by a plethora of Medline abstracts that are now appearing on the net.

What do you do if you want to find infor-

Robert Sikorski is a Howard Hughes Medical Institute fellow at the National Cancer Institute and Richard Peters is in the department of medicine, Massachusetts General Hospital (rss@nchgr.nih.gov; rpeters@vp3.med.harvard.edu; http://www.edsitenavigator.com). mation about a specific knockout or transgenic mouse strain? There are two good places to start, each differing in their layout. A large database collection of knockout mice and transgenics can be found at the Frontiers in Bioscience WWW site. Here, they have taken a directory approach to navigation. An easy to follow design allows you to browse alphabetically or by overall phenotype. The non-expert surfer should start here first. The other good starting place is the TBASE site which has a search engine type of format. The site seems to be quite up-to-date and has an amazing degree of organization. You can search the TBASE data set by any combination of over 25 different data fields (e.g. line name, DNA construct, lab contact, or citation). The "method" field for instance, allows you to search for all mice generated by a specific technique (microinjection, transfection, retrovirus infection, etc.). Before you dive in to do a TBASE search, take a look at the examples page. The search capabilities are powerful, but the format is not very intuitive to the average user. There are a handful of mouse databases on the Net today, and it is difficult to know the completeness of any one particular database. Over time, history shows that such data collections are difficult to maintain. For now, there are several gallant attempts worth investigating. Try out a couple and you are likely to find the information you need.

As always, additional links pertaining to this column can be found online at the YML section of the Medsite Navigator (www.medsitenavigator.com). If we have missed anything, send us your suggestions for the web column.

\section{A sampler of products and services sites related to transgenics}

Frontiers in Bioscience's database http://www.bioscience.org/knockout/knochome.htm of gene knockouts

TBASE: The transgenic/targeted mutation database

Biology of the mammary gland web site

Taconic laboratory

Transgenic discussion page

The Jackson Laboratory

It's a knockout!

Mouse genome database

Whitehead genetic and physical maps of the mouse genome

Oak Ridge transgenic and targeted mutant animal database

Sinai's mammary transgene database

http://www.gdb.org/Dan/tbase/tbase.html

http://mammary.nih.gov:80/

http://www.taconic.com:80/altpages/aanimals/ aproduct.shtml

http://www.jax.org:80/

http://www.gdb.org/Dan/tbase/docs/knockout.html

http://www.informatics.jax.org/mgd.html

http://www.ornl.gov/TechResources/Trans/ hmepg.html mtdb.html http://www.lists.ic.ac.uk:80/hypermail/transgenic-list/ An interesting discussion of transgenic technology

http://www.genome.wi.mit.edu/cgi-bin/mouse/index

http://condor.mbcr.bcm.tmc.edu/BEP/ERMB/ issues from the Imperial College, London.

This database of knockout mice is arranged so that searches can be made based on phenotype or based on the gene involved. The layout is first rate, and the authors have attempted to provide links to gene sequence and literature information as well.

A highly organized and searchable database of mouse knockouts and transgenics.

This is an amazing collection of resources dealing with the mouse mammary gland. The topics range from knockout mice to transgenics to naturally occurring strains.

This page lists several transgenic strains aviailable from this company. In addition, the site has a nice section on general mouse care and techology.

The home site of this company is a must-see for anyone in the field. If you have an interesting strain, look into the online submission section for distributing it through Jackson Labs.

An online review of selected knockout mice is posted every three months by the publishers of Trends in Genetics.

To obtain mouse markers, DNA probes, and maps.

A collection of useful mapping resources from MIT. induced mouse mutations. These data are largely mirrored at the TBASE site.

Another database for those focusing on mammary gland biology.
A highly structured database of information on 